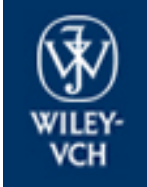

Biotechnology Journal

\title{
Using BRET to study chemical compound-induced disruptions of the p53-HDM2 interactions in live cells.
}

\begin{tabular}{|r|l|}
\hline Journal: & Biotechnology Journal \\
\hline Manuscript ID: & biot.200900272.R1 \\
\hline Wiley - Manuscript type: & Research Article \\
\hline Date Submitted by the \\
Author: & 28-Jan-2010 \\
\hline Complete List of Authors: & $\begin{array}{l}\text { MAZARS, Anne; INSERM, UMRS 940 } \\
\text { Fahraeus, Robin; INSERM, cibles therapeutiques }\end{array}$ \\
\hline Primary Keywords: & Methods \\
\hline Secondary Keywords: & Cancer \\
\hline Keywords: & p53, HDM2, Bioluminescence Resonance Energy Transfer \\
\hline &
\end{tabular}

\section{scholarONE \\ Manuscript Central}




\title{
Using BRET to study chemical compound-induced disruptions of the p53-
} HDM2 interactions in live cells.

\author{
Anne Mazars and Robin Fåhraeus \\ C-DITHEM, INSERM, Paris, France
}

Correspondence: Dr. Robin Fåhraeus, Cibles Thérapeutiques, INSERM UMRS 940, Institut de Génétique Moléculaire, Université Paris 7, Hôpital St-Louis, 27 rue Juliette Dodu, 75010 Paris, France ; robin.fahraeus@inserm.fr; Tel : +33 142499 269, Fax : +33 142494838.

\begin{abstract}
Abbreviations : BRET, Bioluminescence Resonance Energy Transfer; PPI, Protein-Protein Interactions ; Rluc, Renilla luciferase ; YFP, Yellow Fluorescent Protein ; HDM2, Human Mouse Double-Minute 2.
\end{abstract}

Keywords: p53, HDM2, Bioluminescence Resonance Energy Transfer, drug development. 
Abstract

Modification of protein-protein interactions (PPIs) holds promise for novel rational drug design. Disrupting or modifying protein interactions offer new challenges in terms of chemical compound libraries and techniques for compound validation. As proteins interact with several partners in different allosteric conformation in pathological and tissue specific fashions it is difficult to predict the in vivo effect of PPI acting compounds identified by in vitro screening assays. It is therefore desirable to develop techniques that rapidly allow cell-based validation of protein interacting compounds. The binding of the p53 tumour suppressor to the HDM2 E3 ubiquitin ligase is important for controlling p53 activity and several compounds, such as Nutlin-3, have been designed to bind a hydrophobic pocket in the N-terminus of HDM2 to prevent the interaction with p53 in order to stabilize and activate downstream p53 pathways. We have used the p53-HDM2 interaction as a model system to explore the Bioluminescence Resonance Energy Transfer (BRET) technique for validating compounds that disrupt protein-protein interactions in living cells. 


\section{Introduction}

There is an ever increase in the numbers of known protein-protein interactions (PPIs) in both normal and pathological conditions [1]. To specifically control these interactions, using small compounds has the potential of bringing on a new generation of less toxic and more specific therapeutic treatments. As compared to inhibitors of enzyme activity, the development of compounds that target PPIs is more challenging. Apart from identification of the actual chemical compounds that can modify PPIs, most proteins implicated in disease have several interacting partners and it is not always clear which interaction should be targeted to give desired cell biological benefits. Hence, targeting PPIs offers new types of tests for chemistry and target evaluation. Our knowledge on the potential role of a protein in the development of disease is usually derived from studies analysing overexpression, or absence, of the entire protein. For example, overexpression of a kinase in a certain type of cancer gives a clear indication that targeting the catalytic domain might prove useful in treating the cancer. This was the case for targeting the BCR-ABL tyrosine kinase in chronic myeloid leukemia which lead up to the discovery of Imatinib that has been hailed as success story for rational drug design based on basic cancer research [2]. However, information on the expression levels of a protein is not as useful when it comes to targeting a PPI as it cannot be taken for granted that disrupting an interaction with a certain partner will have the same effect as knocking out the entire protein. Hence, knocking out a protein using siRNA, or similar techniques, will not necessarily give the same cell biological effects as disrupting a PPI and, thus, target evaluation of PPIs generally requires more work and different types of analysis. Choosing the most suitable PPI target requires 
good insight into the regulation of the protein complex and the best site for interfering with a PPI is not always obvious. For example, both the tumour suppressor p16 and the p $21^{\mathrm{CDKN} 1 \mathrm{~A}}$ interact with CyclinD/CDK complex and prevent the phosphorylation of the Rb protein and result in a G0 to G1 cell cycle progression arrest but have only partly overlapping cell biological activity and little sequence similarities. This illustrates not only the challenge of evaluating and targeting PPIs but also the great opportunity that modifying PPIs offers in terms of specific cell biological effects. It also suggests that even for a kinase complex such as the Cyclin/CDK, PPI modification can offer different results as compared to catalytic inhibitors. Furthermore, PPIs can involve allosteric interactions that are affected by protein modifications or interacting factors in tissue dependent fashions and disrupting a PPI in vitro using a small compound does not necessary mean that the same PPI will be disrupted in the desired cell type in vivo. All this puts pressure on developing simple and reliable techniques for evaluating PPI modifying molecules and target evaluation in cell-based assays. Ideally, such assays should allow for a first rapid evaluation of a compound or a compound mimetic, before further medicinal chemistry and cell biological tests are initiated.

The use of bioluminescence resonance energy transfer, or BRET, holds promise to fulfill at least some of the criteria for a simple and reliable cell-based assay to test the effect of compounds on disrupting or even modifying a PPI [3-4]. The BRET is based on fusing the Renilla luciferase (Rluc, energy donor) and YFP (acceptor) to either of two interacting proteins. Rluc oxidizes its substrate Coelenterazine $\mathrm{h}$, resulting in the emission of energy. If a suitable energy acceptor is in close proximity, less than $100 \AA$, and is favourably oriented, this energy can be transferred. The excited acceptor molecule then emits energy at a longer wavelength [5]. 
We have used the p53-HDM2 model to evaluate the use of BRET for testing disruption of PPI in living cells. Genetic data has shown that HDM2 is over-expressed in many cancers, mostly sarcomas, and cell-based assays have shown that the binding of the N-terminus of HDM2 with the Nterminus of p53 controls the stability of p53 [6]. HDM2 is an E3 ubiquitin ligase and promotes p53 polyubiquitination but targeting E3 ligase activity is proven difficult and it was postulated that disrupting the p53-HDM2 interaction would be a way of activating p53 in cancers over-expressing HDM2. Several compounds with the capacity to bind the hydrophobic pocket in HDM2 and to compete with p53 binding has been identified, most notably Nutlin-3 [7]. These compounds are based on rational drug design from the co-crystal structure of p53 and HDM2 [6]. 


\section{Material and methods}

\section{Cell culture, transfection and reagents.}

H1299 (human p53-negative non-small lung carcinoma cells) were cultured in RPMI 1640 medium, in the presence of foetal calf serum $10 \%$, L-glutamine $2 \mathrm{mM}$, penicillin $100 \mathrm{U} / \mathrm{ml}$ and streptomycin $100 \mu \mathrm{g} / \mathrm{ml}$ at $37^{\circ} \mathrm{C}$ in a humidified $5 \% \mathrm{CO}_{2}$ atmosphere. Cells were seeded in 6-wells plates at a density of $2 \times 10^{5}$ cells/well. The following day the cells were transfected with $1 \mu \mathrm{g}$ of total expression plasmid along with Genejuice according to the manufacturer's protocol (Novagen). Coelenterazine $\mathrm{h}$ was from Interchim, Nutlin-3 and Cycloheximide were from Sigma-Aldrich.

\section{Plasmid constructs.}

Restriction enzymes, T4 DNA ligase and calf intestinal alkaline phosphatase were obtained from New England Biolabs. Purified synthetic oligonucleotides were obtained from Eurogentec. Rluc-p53 was made using the forward primer 5'- CCGCTCGAGGATGGAGGAGCCGCAGTCAG-3' (restriction sites are in boldface) and reverse primer 5' CGCGGATCCGCGTCAGTCTGAGTCAGGCCC-3' and cloned into pRluc-C1 (BioSignal Packard); YFP-HDM2 was made using 5'- CCGGAATTCCGGATGGCAATACCAACATGTC-3' forward primer and 5'- CGCGGATCCGCGCTAGGGGAAATAAGTTAG-3' reverse primer and cloned into the pEYFP-C1 (Clontech).

Site-Directed Mutagenesis. 
Plasmids coding for Rluc-p53.F19A was prepared using the QuikChange site directed mutagenesis kit (Stratagene), following the instructions of the manufacturer.

\section{Electrophoresis and Western blotting.}

Cells were lysed with $\mathrm{KCl} 100 \mathrm{mM}$, Triton X-100 1\%, HEPES KOH pH7.5 20mM, betaglycerophosphate $50 \mathrm{mM}$, EDTA $1 \mathrm{mM}$, EGTA $1 \mathrm{mM}, \mathrm{Na}_{3} \mathrm{VO}_{4} 0.5 \mathrm{mM}$, glycerol $10 \%$, in the presence of Complete protease inhibitor cocktail (Roche). Protein concentrations were measured using Bradford (BioRad) and separated by 10\% SDS-polyacrylamide gel electrophoresis (PAGE). Membranes were incubated overnight at $4{ }^{\circ} \mathrm{C}$ with polyclonal anti-p53 (CM1) or monoclonal antiMDM2 (4B2) antibodies. The membranes were washed before being incubated with Horse Radish Peroxidase-conjugated rabbit anti-mouse or swine anti-rabbit immunoglobulin antibodies (Dako) and detected using Super Signal® West Pico Chemiluminescent Substrate (Thermo Scientific). Immunoprecipitations were performed on $500 \mu \mathrm{g}$ of cell lysates by using CM1 or 4B2 antibodies. After incubation, co-immunoprecipitated proteins and inputs were analyzed by Western blot.

\section{Immunofluorescence.}

H1299 cells were transfected with the different constructs and observed after 24 hours using a reversed microscope (Axiovert, Zeiss) according to standard methods. Pictures were obtained using a camera (Micromax $5 \mathrm{MHz}$ 1300x1030 pixels) managed by Metamorph software (Princeton Instruments), with 100x/1.4 oil plan Apochromat objective. Cell nuclei were stained with DAPI. HDM2 was observed with 4B2 and anti-mouse/Alexa Fluor®568 (InVitrogen) antibodies, Rluc-p53 was observed with CM1 and anti-rabbit/Alexa Fluor®647 (InVitrogen) antibodies. 


\section{BRET Saturation Assays.}

Cells were seeded on 6-wells plates and transfected with $50 \mathrm{ng}$ per well of the DNA constructs coding for BRET donors and increasing amounts (0.1-950 ng per well) of the BRET acceptor plasmids (or control YFP). $24 \mathrm{~h}$ post-transfection, the luciferase substrate, Coelenterazine h, was added at a final concentration of $5 \mu \mathrm{M}$. Luminescence and fluorescence were measured simultaneously by using the Mithras fluorescence luminescence detector (Berthold). Cells expressing BRET donors alone were used to determine background. Filter sets were $485 \pm 10 \mathrm{~nm}$ for luciferase emission and 530 $\pm 12.5 \mathrm{~nm}$ for YFP emission. BRET ratios were calculated as described [8]. The data show the average values of triplicates from three independent experiments. 


\section{Results}

The p53 and HDM2 interact via respective N-termini. In order to use BRET to study this interaction we fused the Renilla luciferase donor sequence to the N-terminus of p53 (Rluc-p53) and the YFP acceptor to the N-terminus of the human form of MDM2 (HDM2) (YFP-HDM2) (Fig. 1A). To ensure that fusing the Rluc and YFP to respective protein does not affect the sub-cellular localization which, in turn, could affect the BRET, we tested the localization of each fusion protein in p53 null H1299 cells. Figure 1B upper row shows that expression of p53 and HDM2 results in a nuclear localization of both proteins in the majority of cells and with a fraction of cells expressing each protein also in the cytoplasmic compartment. When Rluc-p53 or YFP-HDM2 where expressed, we observed a similar pattern with each protein being expressed in the nucleus of all cells but also in the cytoplasmic compartment in a portion of cells. The expression of the fusion constructs were verified using immunoblotting against specific p53 and HDM2 sera, respectively, or direct visualization of YFP. Next, the interaction between the two fusion constructs was tested using standard immunoprecipitation (IP) assays. Expression of Rluc-p53 and YFP-HDM2 followed by IP of HDM2 revealed the co-IP of Rluc-p53 in a similar fashion as p53 co-IP with HDM2 (Fig. 1C, upper panels). Transfected Rluc-p53 is able to interact with endogenous HDM2. In a similar way, we observed that IP of Rluc-p53 or p53 lead to the co-IP of YFP-HDM2 or HDM2, respectively. These results indicate that Rluc-p53 and YFP-HDM2 interact with each other in a similar way as the non-tagged proteins and that fusion of Rluc and YFP to p53 and HDM2 does not appear to affect the sub-cellular localization. 
We next introduced the BRET reporter constructs into H1299 cells using 6-wells plates. The Rluc-p53 donor encoding plasmid was transfected at a constant amount of $50 \mathrm{ng}$ in all wells and plasmids encoding YFP itself, or YFP-HDM2, were transfected at increasing amounts from 0 to 950 ng. As p53 proteins form oligomers we also co-expressed YFP-p53 as an additional acceptor to Rlucp53 and as a control to the p53-HDM2 BRET. The results show that either YFP-HDM2 or YFP-p53 give rise to a strong specific BRET signal in the presence of Rluc-p53. The BRET 50 value indicates the amount of proteins required to give half of the max BRET and is a direct indicator of the affinity of the donor and acceptor with a low number indicates a higher affinity. The $\mathrm{BRET}_{50}$ was determined to be 1.5 for YFP-HDM2 and Rluc-p53 and 0.9 for the p53 oligomers, indicating that under these conditions the p53 oligomers form more easily, as compared to p53-HDM2 complexes (Fig. 2A).

When we swapped the donor acceptor pairs, or changed the fusion of the donor/acceptors from the $\mathrm{C}$ - to the $\mathrm{N}$-termini, we could confirm BRET between all possible combinations even though the pair in which both donor and acceptor were localized to the N-termini was found to give the highest BRET signal (Fig. 2 and data not shown). The Box I domain of p53 is highly conserved and located between amino acids $15-26$ and binds to a hydrophobic pocket in the N-terminus of HDM2. Mutation of phenylalanine to alanine at position 19 in p53 (p53.F19A) has previously been shown to disrupt the p53-HDM2 interaction and the introduction of F19A in Rluc-p53 abolished the BRET reaction with YFP-HDM2 [9]. Similarly, deletion of the first 68 residues in HDM2 (YFPHDM2(69-490)) which harbors the p53 binding pocket also resulted in a complete inhibition of the BRET (Fig. 2B). These data confirms the specificity of the p53-HDM2 BRET reaction. 
Having established that the p53/HDM2 BRET reporter proteins interact with each other in a similar fashion as the wild type p53 and HDM2, we next set out to test if we can use the BRET assay to study the effects of small compounds on modulating specific protein-protein interactions in live cells. Nutlin-3 is a well-tested compound that binds to the hydrophobic pocket of HDM2 and competes for p53 binding. When $10 \mu \mathrm{M}$ Nutlin-3 was applied to a cell culture expressing Rluc-p53 for eight hours, we could observe a significant decrease in the p53-HDM2 BRET (Fig. 3A). However, there was no significant change to the $\mathrm{BRET}_{50}$ value under these conditions, indicating that the affinity for HDM2 to p53 is not changed in the presence of Nutlin-3, despite the fact that the mBRET is reduced to less than half. This is what is expected of a compound which acts as a competitor. When we instead added Nutlin-3 to cells expressing the YFP-p53 acceptor protein there was no effect, confirming the specificity of Nutlin-3 for the p53-HDM2 BRET assay (Fig. 3A). We next performed a dose-response experiment with Nutlin-3 levels increasing from 1 to $30 \mu \mathrm{M}$ and we could observe a reduction in the BRET up to $10 \mu \mathrm{M}$ whereas there was no further reduction at 30 $\mu \mathrm{M}$, indicating that Nutlin-3 reached saturating levels at $10 \mu \mathrm{M}$ (Fig. 3B). Interestingly, even though the $\mathrm{BRET}_{50}$ does not change much, there is still a specific BRET at $30 \mu \mathrm{M}$, indicating that p53 and HDM2 still interact, presumably through the reported second interface site [10].

We also tested the effect of Nultin-3 on the p53-HDM2 complex over time. Addition of 10 $\mu \mathrm{M}$ Nutlin-3 for one to eight hours resulted in a gradual decrease in the mBRET without affecting the levels of Rluc-p53 or YFP-HDM2 notably (Fig. 3C). This delay in efficacy is unlikely due to a delay in the uptake of Nutlin-3 but instead indicates that Nutlin-3 is more either effective in preventing the formation of new p53-HDM2 interactions, as compared to disrupting already established ones, or that 
the complexes are stable. To further address this possibility we treated cells for two hours with the ribosomal inhibitor cyclohexamide $(\mathrm{CHX})$. This will stop protein synthesis and, thus, changes in the mBRET will be due to disruption of established old complexes. However, treatment with CHX had no additional effects as compared with Nutlin-3 alone (Fig. 3D).

\section{Discussion}

The manipulation of protein-protein interactions forms an interesting and challenging objective for novel therapeutic drug development. This is a yet relatively unexplored area of therapeutics but there are examples of drugs under development that specifically targets PPIs and some compounds with known effects are found to be acting via modifying PPIs. For example, brefeldin A, a potent inhibitor of the Golgi secretion pathway, acts by allosteric interference with the small G-protein Arf1 [11]. Similarly it is believed that rapamycine, which is an inhibitor of mTOR, acts via allosteric mechanisms [12]. It is clear that successful manipulation of PPI holds promise for more specific and targeted therapies and it is therefore important to develop new tools and methods for better studying compounds that can manipulate PPIs. This might include different types of chemical libraries than those used for identifying enzyme catalytic inhibitors and also techniques for identifying PPI targeting compounds in living cells as well as studying the effects of PPI manipulating compounds. A PPI is dependent on a number of different factors. Post translational modifications can induce direct allosteric changes in one or both of the partners, or promote the binding of a third factor to the complex. More often than not does a target protein of interest interact with more than one partner and, hence, the multi protein complexes found within the cells are different as compared to that of 
two recombinant purified proteins interaction in vitro. It is uncertain that compounds identified in vitro will have desired effect in cells, neither in terms of PPI interfering activity as well as cell biological effects. Hence, targeting PPIs requires, to a higher degree than for catalytic inhibitors, the access to in cellulo assays early on in the compound identification process in to rapidly evaluate in vitro derived compounds. Another aspect of our work is to develop cell-based assays to identify PPI active compounds for small to medium size screening assays.

Based on this reasoning, we have tested if the BRET assay can be developed to satisfy these demands. We have used the well-established p53-HDM2 interaction and the equally well-described Nutlin-3 molecule. By carefully comparing the interaction between HDM2 and p53 BRET constructs with that of the non-tagged wild type proteins we believe the interaction between the BRET constructs and the wild type proteins are similar. Point mutation in the Box I of p53, that is known to prevent the interaction with HDM2, also prevents the Rluc-p53 interaction with YFP-HDM2. Deletion of the N-terminus of HDM2 is equally efficient in preventing the interaction. The capacity of Nutlin-3 to suppress the binding of HDM2 to p53 BRET constructs, but not the p53-p53 constructs, further underlines the specificity of the interaction. We observed that $10 \mu \mathrm{M}$ of Nutlin-3 was the maximum concentration with which the mBRET for p53-HDM2 could be inhibited. Interestingly, this did not completely prevent the p53-HDM2 interaction. It has been reported that p53-HDM2 consists of two interfaces [10]. The first is the well-known N-termini target for Nutlin-3 in which the Box I domain of p53 fits into a hydrophobic pocket of HDM2. But there is also an interaction involving more central domains which is activated by molecules binding the hydrophobic N-terminal pocket of HDM2, including p53 itself and Nutlin-3. It is thus, possible that this second 
interface is what remains after treatment with high concentrations of Nutlin-3. What is a bit surprising, if this indeed is the case, is that the affinity of this interaction appears similar as that of the $\mathrm{N}$-termini interaction. The fact that the mBRET is lower can simply imply that the positioning of the donor acceptor is less favorable and further apart when the second site is occupied and does not necessarily mean that there are less interacting complexes.

We did not observe major differences in the BRET $_{50}$ of the p53-HDM2 complex using Nutlin3, even though the overall mBRET was significantly reduced. As the BRET ${ }_{50}$ indicates the affinity of the donor-acceptor this is what one would expect by a competitive inhibitor such as Nutlin-3. We have not yet been able to test the BRET for compounds that induce changes in PPIs via allosteric mechanisms. It would be interesting to see if it is possible to use the BRET to indicate if PPI modifying compounds act via allosteric mechanism or via direct interface competition. This is something we will be looking into further. It can be mentioned that when we have carried out BRET using p53 as both donor and acceptor we have identified compounds that increases p53 oligomerisation and changes the BRET $_{50} 10$-fold (unpublished results).

We performed a time course with Nutlin-3 at $10 \mu \mathrm{M}$ concentration and we observed a decrease of the mBRET point over time. There is little reason to expect that the uptake of Nutlin-3 is delayed in these cells and Western blot analysis show that this reduction is not due to a corresponding decrease in the levels of the donor or acceptor. This latter would have resulted in a shift in the concentration of acceptor required to reach max mBRET, rather than in an overall decrease, which we did not observe. It should be pointed out that HDM2-dependent degradation of p53 is not very effective in the H1299 cells used for these assays and the half life of p53 is much higher than the 20 
minutes reported in some conditions. We also observed that the mBRET of p53-HDM2 was not affected by inhibitor of protein synthesis. These results are not conclusive but might indicate that the time-dependent effect of Nutlin-3 on the p53-HDM2 is not due to preventing complex formations vs. disrupting existing complexes. Thus, the reduction in mBRET over time might indicate that the complex is quite stable under these conditions and that the nutlin would need quite a long time to be able to disrupt existing complexes. It is, of course, possible that this is a phenomenon related to the BRET constructs and does not apply to the endogenous p53-HDM2 interaction. Nevertheless, as a part of investigating the use of BRET for intracellular PPI drug evaluation these results suggest that compound stability might be an important factor even during early steps in a cell-based evaluating process of potential PPI active compounds.

The advantage of BRET for these types of assays is that it is remarkable robust and reproducible. Under these conditions we have used transiently transfected cells for both the donor and the acceptor constructs and we found that the results are very similar from one experiment to the next. It is possible, however, that the establishment of stable cell lines for one, or both of the BRET constructs, will further increase the reproducibility. This is most likely also the way forward for standardizing assays and for developing the BRET for screening purposes. In these studies we have used 6-wells plates but we have also found that up to 24-wells plates work fine. Going to smaller size wells will most likely require stable transfected cell lines. This is currently being developed.

The use of Nutlin-3 nicely demonstrates how a PPI can be monitored in live cells by modifying compounds. This technique should allow compounds derived from in vitro-based high throughput assays to be rapidly tested in cells and could greatly reduce the amount of work needed to evaluate the effect of compounds destined for drug development. 


\section{Acknowledgments}

This work is funded by the INSERM C-DITHEM platform and La Ligue Contre le Cancer. We thank Cédric Boularan and Stefano Marullo (Institut Cochin) for help with BRET, Borek Vojtesek (Masaryk Memorial Cancer Institute, Brno, Czech Republic) for p53 and HDM2 antibodies, Guy Millot (INSERM UMRS 940) for his help in fluorescence microscopy.

\section{References}

[1] van Montfort, R. L. and Workman, P., Structure-based design of molecular cancer therapeutics. Trends Biotechnol 2009, 27, 315-328.

[2] Druker, B. J., Talpaz, M., Resta, D. J., Peng, B., et al., Efficacy and safety of a specific inhibitor of the BCR-ABL tyrosine kinase in chronic myeloid leukemia. N Engl J Med 2001, 344, 1031-1037.

[3] Milligan, G., Applications of bioluminescence- and fluorescence resonance energy transfer to drug discovery at G protein-coupled receptors. Eur J Pharm Sci 2004, 21, 397-405.

[4] Bacart, J., Corbel, C., Jockers, R., Bach, S. and Couturier, C., The BRET technology and its application to screening assays. Biotechnol J 2008, 3, 311-324. 
[5] Kasprzak, A. A., The use of FRET in the analysis of motor protein structure. Methods Mol Biol 2007, 392, 183-197.

[6] Kussie, P. H., Gorina, S., Marechal, V., Elenbaas, B., et al., Structure of the MDM2 oncoprotein bound to the p53 tumor suppressor transactivation domain. Science 1996, 274, 948-953.

[7] Vassilev, L. T., Vu, B. T., Graves, B., Carvajal, D., et al., In vivo activation of the p53 pathway by small-molecule antagonists of MDM2. Science 2004, 303, 844-848.

[8] Storez, H., Scott, M. G., Issafras, H., Burtey, A., et al., Homo- and hetero-oligomerization of beta-arrestins in living cells. J Biol Chem 2005, 280, 40210-40215.

[9] Yin, Y., Stephen, C. W., Luciani, M. G. and Fahraeus, R., p53 Stability and activity is regulated by Mdm2-mediated induction of alternative p53 translation products. Nat Cell Biol 2002, 4, 462-467.

[10] Wallace, M., Worrall, E., Pettersson, S., Hupp, T. R. and Ball, K. L., Dual-site regulation of MDM2 E3-ubiquitin ligase activity. Mol Cell 2006, 23, 251-263.

[11] Pommier, Y. and Cherfils, J., Interfacial inhibition of macromolecular interactions: nature's paradigm for drug discovery. Trends Pharmacol Sci 2005, 26, 138-145. 
[12] Berg, T., Inhibition of transcription factors with small organic molecules. Curr Opin Chem Biol 2008, 12, 464-471.

\section{Figure legends}

Figure 1.

A) Cartoon illustrating the p53 and HDM2 BRET assay constructs. The position of the Renilla Luciferase donor (Rluc) and Yellow Fluorescent acceptor Protein (YFP) are indicated together with some key domains of respective protein. The p53-HDM2 interaction is mediated by the conserved Box 1 domain of p53 (HDM2 BD) and the hydrophobic pocket of HDM2 (p53 BD). TAD stands for transactivation, PxxP for polyproline rich and $\mathrm{T}$ for tetramerization domain, respectively. B) Immunofluorescence reveals the sub-cellular localization of p53 and HDM2 using the polyclonal CM-1 anti-p53 sera or the 4B2 anti-HDM2 mAb, respectively. DAPI staining shows the cell nucleus. The sub-cellular localization of the YFP acceptor BRET construct is detected using indicated wavelength. The figure shows that the BRET constructs and the non-tagged proteins can be found in the nucleus only or in the cytoplasmic and nuclear compartments. C) Transient expression of indicated BRET or non-tagged constructs followed by immunoprecipitation (IP) and Western blot (WB) shows the interaction of each protein complex. CE stands for Crude Extracts.*Indicates endogenous HDM2. The results show data representative of three independent experiments. 
Figure 2.

\begin{abstract}
A) BRET assay using increasing amounts of vectors encoding YFP alone, or YFP-p53 and YFPHDM2 acceptors in the presence of a fixed amount of the vector encoding Rluc-p53. The BRET 50 is half max mBRET and indicates the affinity of the donor acceptor pair. As p53 forms oligomers and interacts with HDM2 both the YFP-p53 and the YFP-HDM2 acceptors give a BRET in the presence of the p53RLuc donor. B) Introducing the F19A mutation in p53 or deletion of the HDM2 Nterminus including the p53-BD, prevent the p53-HDM2 interaction and abolishes the BRET. The results show the average data of triplicate BRET assays from three independent experiments.
\end{abstract}

Figure 3.
A) The p53-HDM2 and the p53-p53 BRET in the presence of $10 \mu \mathrm{M}$ Nutlin-3 for 8 hours. Only the p53-HDM2 interaction is affected. B) Increasing amounts of Nutlin-3 disrupt the p53-HDM2 interaction up to $10 \mu \mathrm{M}$. C) $10 \mu \mathrm{M}$ Nutlin-3 was added at different time points before the BRET was estimated. Western blot analysis shows expression levels. D) BRET assay using a single point corresponding to $\mathrm{BRET}_{\max }$ in figure 3C. Cells treated with the ribosomal inhibitor cyclohexamide (CHX), Nutlin-3 alone, or CHX and Nutlin-3, for a further two hours. The results show data representing three independent experiments plus SD. 
Figure 1

A

Donor: Rluc-p53

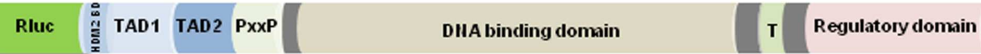

Acceptor:YFP-HDM2

\begin{tabular}{|l|l|l|l|}
\hline YFP & P53 BD & Acidic Domain & Zing finger domain \\
\hline
\end{tabular}

B

p53 + HDM2
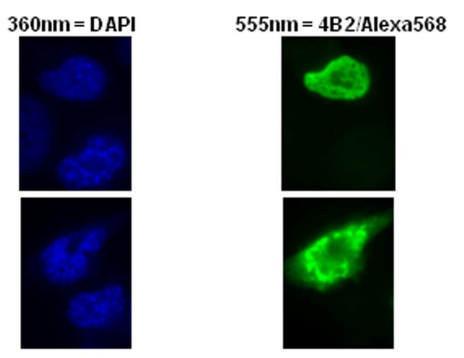

$640 \mathrm{~nm}=\mathrm{CM1} / \mathrm{Alexa} 647$

merge

Rluc-p53 + YFP-HDM2
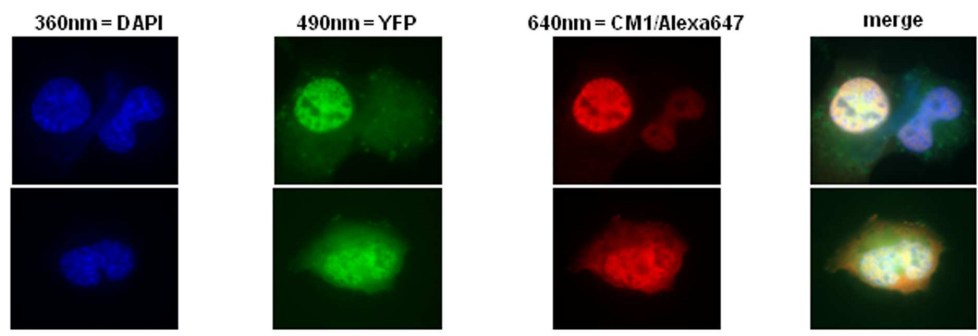

$190 \times 254 \mathrm{~mm}(300 \times 300$ DPI $)$ 
Figure 1

C

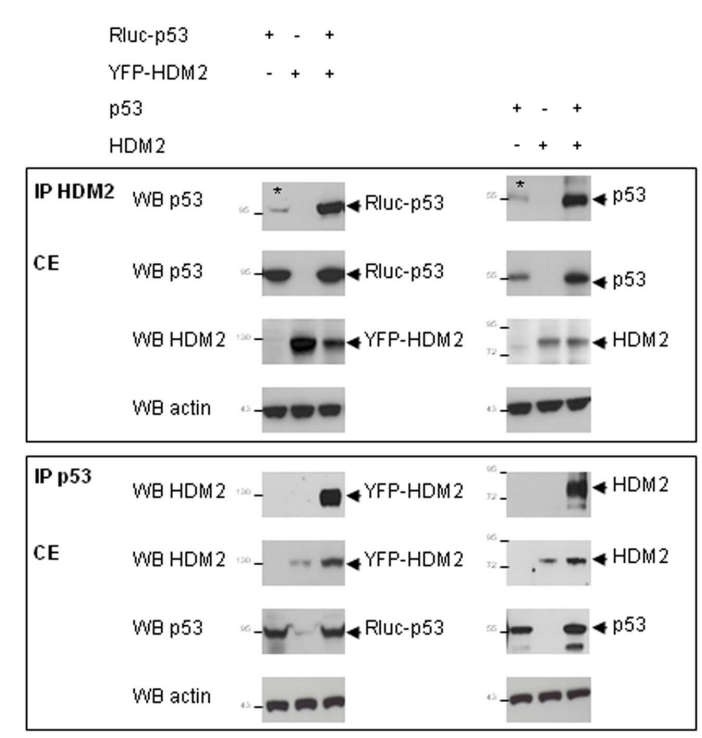

$190 \times 254 \mathrm{~mm}(300 \times 300$ DPI $)$

Wiley-VCH 
Figure 2

A

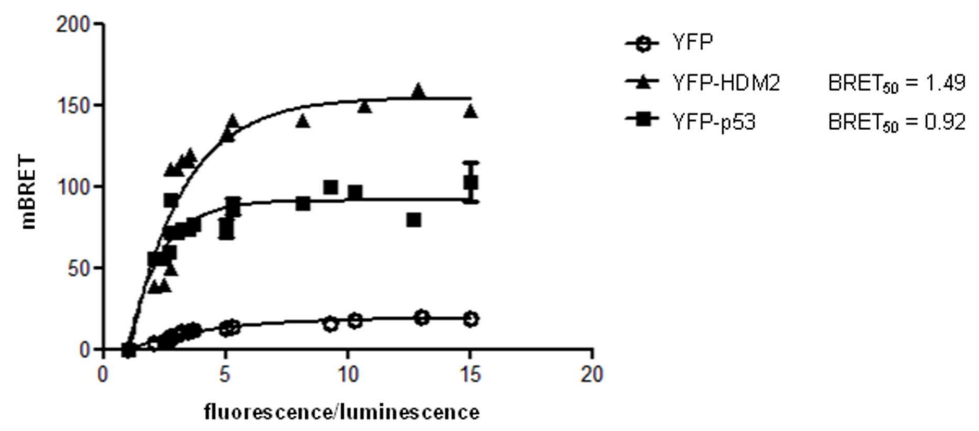

B

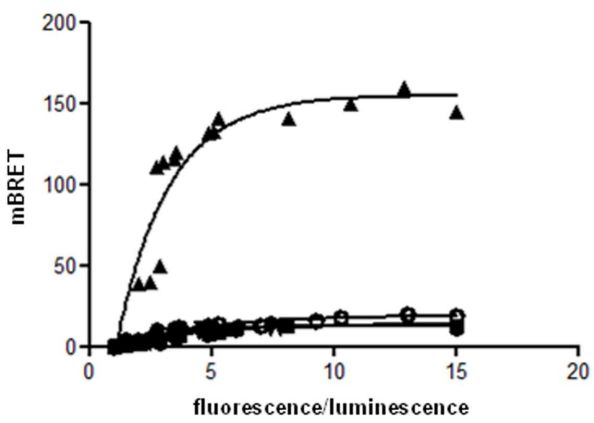

- Rluc-53 + YFP

‡ Rluc-p53 + YFP-HDM2

$\Rightarrow$ Rluc-p53 + YFP-HDM2(69-490)

$\rightarrow$ Rluc-53.F19A + YFP

$\bullet$ Rluc-p53.F19A + YFP-HDM2

$190 \times 254 \mathrm{~mm}(300 \times 300 \mathrm{DPI})$

Wiley-VCH 
A

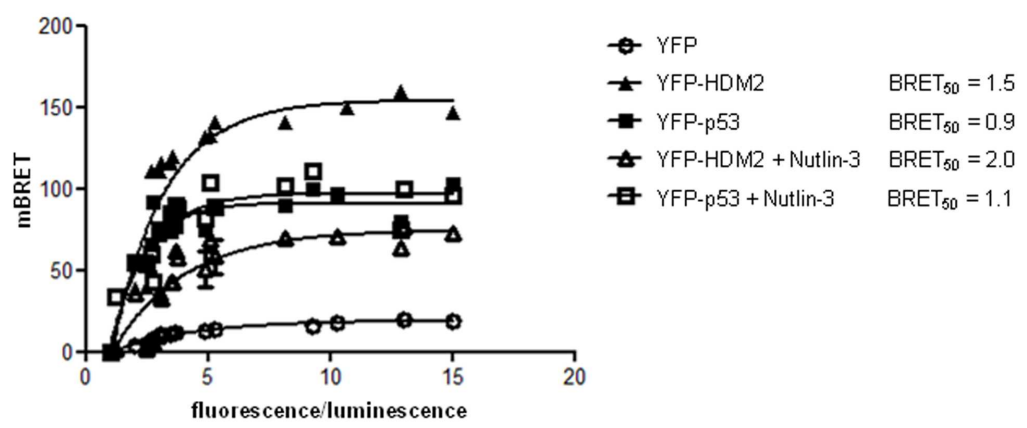

B

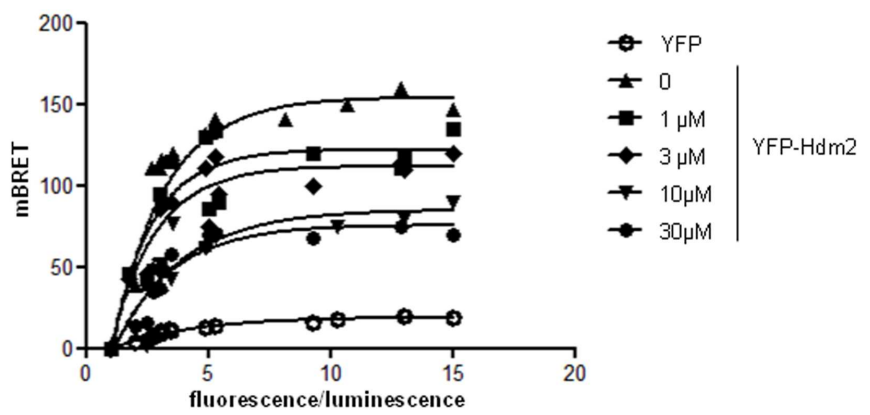

$190 \times 254 \mathrm{~mm}(300 \times 300 \mathrm{DPI})$ 
Figure 3

A

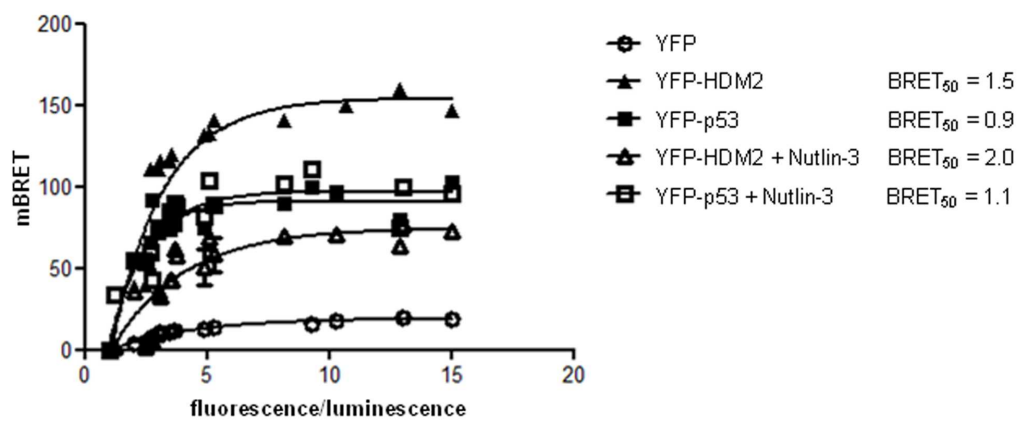

B

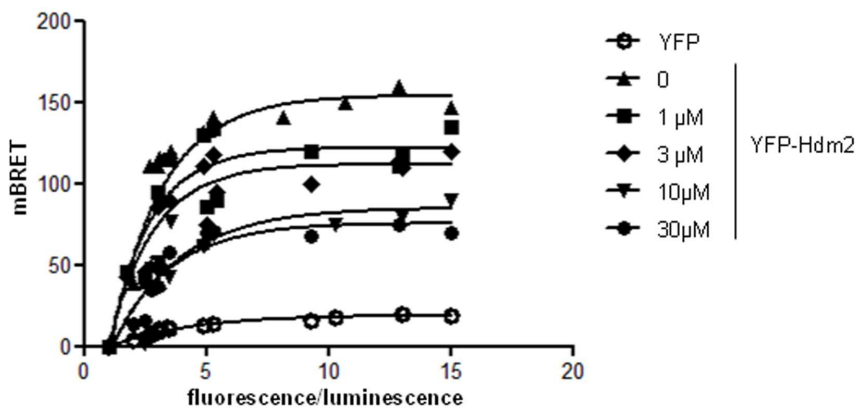

$190 \times 254 \mathrm{~mm}(300 \times 300 \mathrm{DPI})$

Wiley-VCH 